\title{
La liberté des Modernes à l'épreuve de la finitude
}

\author{
Augustin Fragnière \\ Doctorant en philosophie politique, Université de Lausanne, IPTEH, 1015 Lausanne, Suisse
}

Le projet d'Augustin Fragnière est de montrer l'impact de la pensée écologique sur la philosophie politique au fondement du libertarisme et de la propriété privée. L'auteur montre bien en quel sens la «finitude » des ressources rend pertinente cette critique de la philosophie. L'étude présentée dans ce numéro porte surtout sur la philosophie de Locke et ses interprétations contemporaines. Elle est une première étape pour mettre en balance certains résultats de l'écologie et leurs effets sur la pensée philosophique.

La Rédaction

\section{Mots-clés :}

environnement ; finitude ;

liberté ;

droits de l'homme ; philosophie politique

\section{Keywords:}

environment;

finiteness;

liberty;

human rights;

political philosophy
Résumé - Les problèmes environnementaux et les réponses qui pourraient leur être apportées font-ils peser une menace nouvelle sur la liberté individuelle? La question mérite d'être posée à l'heure où l'on prend de plus en plus conscience des limites de la planète, tant au niveau des ressources que de la capacité de charge des écosystèmes. La conception moderne de la liberté, telle que définie par le projet libéral, est faite d'indépendance, de «jouissance paisible » et de droit à la poursuite de son intérêt personnel. Mais estelle compatible avec la finitude de la planète et l'impossibilité qui en découle de poursuivre une croissance universelle des flux de matière et d'énergie? Cet article tente de dégager les tensions qui apparaissent entre le concept libéral de la liberté (hérité de Hobbes et de Locke), ainsi que ses développements libertariens, et la question environnementale, sous deux aspects : celui du progrès technique et celui de la propriété privée.

\begin{abstract}
The liberal concept of liberty versus the finiteness of the planet. Are the environmental problems and the solutions that could be provided posing a new threat to individual freedom? The question may well be asked as we grow increasingly aware of the limits of our planet in terms of resources as well as of carrying capacity of ecosystems. The modern conception of liberty, as defined by the liberal theory, rests on "peaceful enjoyment and private independence" as well as on the pursuit of one's very personal interest. Yet is this kind of freedom compatible with the finiteness of the planet and the impossibility that derives from it to carry on with a universal growth of energy and material flows? This article shows the tensions arising between the classic liberal concept of liberty (inherited from Hobbes and Lock), and its libertarian developments, and the environmental issue, regarding technological advancement and private ownership. The former is increasing our capacity for action, which increases potential damages to the environment, while the liberal conception of liberty opposes any restriction to the use of new technologies. The latter is intrinsically linked to the concept of liberty, understood as the right to unlimited accumulation of wealth. Human rights have always been the theatre of various contradictions. Introducing the environmental issue into the debate creates a further contradiction that will most certainly lead to a reinterpretation of these rights.
\end{abstract}

Les problèmes environnementaux et les réponses qui pourraient leur être apportées font-ils peser une menace nouvelle sur la liberté individuelle? La question mérite d'être posée à l'heure où la finitude de la planète s'élève en rempart, de manière chaque année plus évidente, contre une tendance haussière de la consommation mondiale que l'on avait crue éternelle ${ }^{1}$. La finitude de plus

Auteur correspondant : augustin.fragniere@unil.ch

1 Le présent article est le résultat de réflexions menées à l'occasion du colloque Normes et environnement, qui s'est tenu à l’Université de Lausanne les 3 et 4 février 2010. en plus prégnante des ressources d'énergie fossile, des stocks de ressources minérales (métaux précieux et semiprécieux tels qu'or, argent, cuivre, palladium, etc.) et de la capacité de charge du système Terre (changement climatique, dégradation des services écologiques, etc.) rend en effet palpable de manière presque dramatique l'impossibilité d'universaliser le mode de vie occidental et de poursuivre une croissance soutenue des flux de matière et d'énergie ( $c f$. Lebeau, 2008; Rockström et al., 2009; Bourg, 2009; Bourg et Whiteside, 2009 et 2010; Grosse, 2010). Le terme de finitude renvoie donc ici à une finitude 
matérielle. Elle est intimement associée aux limites auxquelles est assignée l'exploitation par les humains de la biosphère (que la science économique en particulier avait pu croire prolonger indéfiniment) en raison du stock fini de ressources dont elle est porteuse et de la fragilité de ses services de régulation. Mais il désigne aussi une finitude toute humaine comprise comme limite à la puissance d'action d'homo faber. Finitude du monde et finitude humaine dialoguent, puisque les problèmes environnementaux sont aussi la marque de la difficulté des humains à prendre en compte sur le plan global les conséquences de leurs actions. Le sentiment de puissance quasi illimitée qu'ont offert aux humains les réussites techniques du $X X^{\mathrm{e}}$ siècle s'estompe désormais peu à peu face aux crises environnementales répétées.

Ces deux formes de finitude interrogent de manière pressante les limites du concept de liberté individuelle hérité du libéralisme classique. La question peut être formulée de la manière suivante : Un retour à une prise en compte accrue des limites imposées à l'agir humain par la finitude de la planète constitue-t-il un retour en arrière en termes de liberté? Et, partant, la traduction politique de cette prise en compte des limites, sous forme de nouvelles normes techniques et comportementales, constitue-t-elle une violation de l'un des principes les mieux affirmés de nos démocraties libérales?

La question n'est à vrai dire pas nouvelle et différents auteurs s'y sont déjà attelés, quelle que soit par ailleurs l'option politique qu'ils défendent. L'autoritarisme assumé de Jonas (1990), par exemple, avec sa «tyrannie bienveillante et bien informée », affirme clairement la nécessité d'une limitation de la liberté individuelle. À l'opposé, d'aucuns dénoncent dans les législations environnementales naissantes l'amorce d'une nouvelle servitude ( $c f$. notamment Ferry, 2002), position parfois accompagnée d'un optimisme scientifique pensant pouvoir résoudre la nouvelle donne planétaire par une réorientation intelligente des techniques vers plus de sobriété. L' « écologie politique », quant à elle, fait de la liberté son maître mot en condamnant à la fois le système productiviste occidental et les risques de dérive «technofasciste » ou «expertocratique » de la démocratie, la solution se trouvant dans une révolution commune de notre organisation politique et du système technique (Illich, 2003; Gorz, 1978) ${ }^{2}$.

\footnotetext{
${ }^{2} \mathrm{~L}^{\prime}$ «écologie politique » désigne ici le courant de pensée porté dans les années 1970 par la pensée d'Ivan Illich, André Gorz (sous le pseudonyme de Michel Bosquet) et Cornélius Castoriadis principalement. Il est à noter que la conception de la liberté sous-tendue par les écrits de ces auteurs est bien différente de la liberté des libéraux, qui est conçue - nous le verrons comme un droit à la poursuite de son intérêt personnel, souvent assimilé à l'appropriation matérielle. Pour les penseurs de l'écologie politique, en revanche, la liberté relève soit de l'autonomie de l'individu libéré de l'aliénation des techniques et du marché (sens négatif), soit de l'autonomie de la société qui
}

Mais, entre un optimisme technique sans nuance, souvent accompagné d'un rejet catégorique de toute forme de normativité écologique, un autoritarisme revendiqué et la nécessité d'un bouleversement total de notre mode de fonctionnement, reste la voie médiane d'une adaptation de la démocratie libérale aux contraintes naturelles. Cette voie, incarnée par le développement durable dans sa variante forte, prend cependant elle-même la mesure de l'immensité des enjeux écologiques et de la profondeur des changements à apporter pour éviter le pire. Le découplage tant attendu entre la création de richesse et la consommation de matière et d'énergie ne s'est pas concrétisé ces vingt dernières années (Jackson, 2010). Devant ce constat, une réflexion sur les principes et l'organisation même des démocraties représentatives modernes, à la lumière des problèmes environnementaux, prend toujours plus de vigueur (The Good Society, 2008; Jancovici et Grandjean, 2009; Longeaux, 2009; Hulot et le comité de veille écologique, 2009 ; Rosanvallon, 2010 ; Gauchet, 2010). Dans ce cadre, une réflexion de fond sur la liberté, en tant que valeur centrale des systèmes politiques occidentaux, et sur sa signification dans le cadre d'une "démocratie écologique » (Bourg et Whiteside, 2010) devient alors indispensable. Afin de répondre à la question posée plus haut - l'adoption de nouvelles normes techniques et comportementales est-elle nécessairement liberticide? - et pour bien cerner les limites imposées à la liberté des Modernes par la nouvelle donne environnementale, il est nécessaire de plonger plus avant dans les différentes conceptions de la liberté qui ont été développées jusqu'à présent par les philosophes. Sans prétendre aboutir à une réponse définitive, les pages qui suivent entendent examiner la conception de la liberté qui se trouve au cœur de la doctrine libérale classique et de son développement libertarien. Des auteurs tels que Thomas Hobbes ou John Locke ont donné le point de départ, il y a trois siècles et demi, de controverses intellectuelles qui perdurent encore aujourd'hui. L'un est à l'origine du concept de liberté tel qu'il est le plus souvent compris de nos jours, l'autre nous a transmis une justification de la propriété privée dont les courants néolibéraux actuels se revendiquent. Les problèmes environnementaux viennent cependant remettre en question certains des présupposés sur lesquels leurs théories étaient fondées. C'est la raison pour laquelle un retour sur ces auteurs anciens est nécessaire pour bien comprendre la nature des liens qui relient la question environnementale et celle de la liberté.

J'entends examiner ici le concept de liberté négative dans ses relations à deux éléments structurants de nos démocraties : le progrès technique et le droit de propriété compris comme droit à l'accumulation illimitée. Ceux-ci entretiennent en effet des relations particulières

se donne à elle-même sa propre loi et ses propres limites (sens positif) [Felli, 2008]. 
à la finitude dans la mesure où, associés à la liberté, tous deux participent d'une logique de la transgression : dépassement de la finitude humaine dans le premier cas; dépassement de la finitude de la nature dans le second. Cet article se borne à analyser les rapports libertéenvironnement d'un point de vue conceptuel. S'il conclut à une tension entre le concept de liberté hérité des libéraux et la situation de crise environnementale vers laquelle l'humanité se dirige, cela ne saurait concerner que les libertés qui engagent des paramètres matériels et qui ont un impact physique sur le monde. Les libertés plus immatérielles telles que liberté de pensée, d'expression ou de conscience ne sont pas directement interrogées par les problèmes écologiques.

\section{Technique et liberté}

Dans leur article «Pour une démocratie écologique », Bourg et Whiteside (2009) montrent en quoi le projet technicien moderne correspond à une tentative d'émancipation de toutes contraintes quelles qu'elles soient, fondée sur la rupture métaphysique qui marque le début de l'ère moderne. Au cosmos fermé et fini de la Grèce antique correspondait une métaphysique de la finitude et une action humaine bornée de fait; à l'univers ouvert et infini de la science moderne correspond l'infinité du progrès et de l'agir humain. «La démocratie moderne est [.. . ] inséparable d'un effort d'arrachement à la tutelle d'un dieu infini et tout-puissant, au sein d'un cosmos désormais muet et insensé. [...] Elle ouvre le désir humain comme l'action technique à l'infini. » « [...] l'action humaine y est appelée à transgresser toutes les limites. » (Ibid.). Tout au long d'une tradition s'étendant de la chrétienté latine à nos jours, trouvant ses premières formulations explicites à l'aube de l'époque moderne avec Francis Bacon, le progrès technique a été l'auxiliaire privilégié de ce vaste projet d'émancipation et de libération de l'humain. Pensons, par exemple, à la jouissance, "sans aucune peine, des fruits de la terre et de toutes les commodités qui s'y trouvent » que Descartes (2000, p. 99) promet par « l'invention d'une infinité d'artifices ». Or, si cette thèse a eu ses contempteurs, à commencer par Rousseau (1996) - mais pensons aussi à Heidegger, Ellul, Illich (2003) ou, plus récemment, Lebeau (2005) -, il est possible de trouver tout au long de l'ère moderne un consensus assez large en faveur de la technique comme créatrice de liberté individuelle. En affirmant l'égale dignité de tous les humains, dignité devant se réaliser par l'accès à la liberté pour tous, la modernité voit dans la maîtrise de la nature par le progrès technique le moyen d'assurer cet accès universel à la liberté. Cette émancipation ne dégage pas seulement du temps libre, elle change aussi la condition d'une partie de l'humanité, qui est désormais déchargée non seulement des tâches matérielles, mais surtout de la domination d'un maître (Bourg, 1997) ${ }^{3}$.

Cette modification radicale de la condition humaine s'accompagne d'une modification tout aussi radicale de la manière de penser la liberté. La liberté d'un citoyen grec était contenue tout entière dans sa participation à la vie publique et à la délibération politique. Comme le rappelle Benjamin Constant (1997), il s'agissait d'une liberté collective compatible avec la soumission totale de l'individu à la collectivité. À l'inverse de cette liberté des Anciens, la liberté des Modernes est faite de «jouissance paisible» et «d'indépendance privée» (ibid., p. 602). Cette conception individuelle de la liberté a conditionné une grande partie du champ de la philosophie politique moderne et contemporaine.

Depuis les premiers travaux d'Isaiah Berlin en 1958, le débat sur la liberté est structuré par une distinction entre deux concepts incommensurables : la liberté négative, qui est grossièrement absence de contrainte, et la liberté positive, qui est maîtrise de soi ou capacité $\mathrm{d}^{\prime}$ autodétermination. Les penseurs libéraux des XVII ${ }^{\mathrm{e}}$ et XVIII ${ }^{e}$ siècles concevaient la liberté d'un point de vue négatif, comme un espace borné au sein duquel l'individu peut mener sa vie comme il l'entend. De ce point de vue, une technique me permettant, par exemple, de parler en temps réel à une personne située à plusieurs centaines de kilomètres de distance met bien à bas une des contraintes qui bornent mon action. On devrait donc pouvoir dire qu'elle augmente ma liberté. Un tel accroissement serait donc éminemment transgressif, puisqu'il vise à faire tomber une à une les limites qui s'opposent à mon action.

Mais il faut ici y regarder de plus près et s'intéresser aux deux dimensions de ces limites : leur source et leur type. La source d'une contrainte peut être naturelle, comme par exemple une rivière impossible à traverser, ou d'origine humaine, comme une loi ou la séquestration d'un individu par un autre. Le type de la contrainte peut être externe, à l'instar d'un obstacle physique, ou interne, dans le cas d'une maladie invalidante ou d'une manipulation idéologique. Pour Berlin (1988, p. 171), les contraintes naturelles doivent clairement être écartées de la définition de la liberté : « La contrainte implique l'intervention délibérée d'autrui dans l'espace à l'intérieur duquel je pourrais normalement agir. [...] Mais la simple incapacité d'atteindre une fin ne constitue pas un manque de liberté politique. »

\footnotetext{
${ }^{3}$ Ce remplacement de la force de travail humaine par la puissance technique a si bien réussi que son existence passe souvent inaperçue aux yeux de la plupart de nos contemporains. Jancovici et Grandjean (2006) l'illustrent de manière amusante en imaginant une nouvelle unité énergétique qu'ils nomment «l'équivalent esclave». Ainsi, d'après leurs calculs, la quantité d'énergie utile au service d'un Européen moyen est la même que s'il possédait en permanence 100 esclaves à son service.
} 
Berlin semble en cela suivre Hobbes (2000, pp. 336$337)$, pour qui, « si l'obstacle au mouvement est la constitution de la chose elle-même, on n'a pas l'habitude de dire qu'elle manque de liberté, mais de puissance de se mouvoir, comme quand une pierre reste là ou que l'on est cloué au lit par la maladie ». Si, par ailleurs, Hobbes ne nie pas l'existence d'obstacles naturels, il montre cependant clairement que «la liberté des sujets réside [...] uniquement en ces choses que, dans le règlement de leur action, le souverain s'est abstenu de prendre en compte » (ibid., p. 340). Il convient donc de bien distinguer ce qui est de l'ordre du manque de liberté et ce qui relève de l'incapacité d'agir (Skinner, 2002). La liberté semble être une notion toute sociale et politique pour ces auteurs et en tous les cas inséparable des interactions humaines. De même chez Locke (1994, § 57 et § 4), la liberté consiste à " être délivré de la contrainte et de la violence exercées par autrui » et l'état de nature est «un état où [les hommes] ont la parfaite liberté d'ordonner leurs actions et de disposer de leurs possessions et de leurs personnes comme ils l'estiment convenable, à l'intérieur des limites de la loi de nature $[. .]^{4}$ ». Nous avons donc chez les fondateurs de la philosophie politique moderne un état de nature, soumis au régime de la nécessité et régi par la loi de la nature, dans le cadre duquel le concept de liberté ne revêt aucune forme de pertinence ${ }^{5}$. Dans cet état, les limites posées à l'action de l'individu isolé, hors de toute interaction humaine, ne sont que le fait de sa nature propre et de celles du monde qui l'entoure. Elles ne constituent donc pas une restriction à sa liberté, mais bien à sa capacité d'action. L'individu à l'état de nature ne peut être dit «parfaitement libre » que par référence à un autre état fait d'interférences entre individus. Cet autre état, social ou présocial, est quant à lui régi par le couple contrainte/liberté.

Si l'on s'en tient à la lettre de cette conception originelle de la liberté négative, les conclusions à tirer sont claires. Le fait de ne pouvoir franchir un océan ou de ne pouvoir défricher une forêt à mains nues ne constitue pas un manque de liberté. Comme le dit si bien Helvétius : «[...] ce n'est pas un manque de liberté que de ne pouvoir voler comme un aigle ou nager comme une baleine» (cité par Berlin, 1988, p. 172). Les moyens auxiliaires permettant à l'homme de réaliser ce qui, a priori, n'est pas dans sa nature ne font donc qu'élargir sa capacité d'action et non sa liberté. De ce point de vue, on ne peut alors pas dire de la technique qu'elle est libératrice. Il y

4 La loi de la nature est ici un principe de conservation qui interdit à l'homme de se détruire lui-même.

5 Si Hobbes reconnaît la validité du terme «liberté » dans le milieu naturel (et c'est d'ailleurs pour lui le seul vrai sens du mot liberté), il s'empresse cependant de préciser qu'il ne l'utilisera que dans un sens politique en référence aux « chaînes artificielles » inventées par les hommes, ce qu'il nomme la « liberté des sujets » (Skinner, 1991). a fort à parier que nos grands-parents ne se plaignaient pas d'un manque de liberté du fait de ne pas pouvoir se rendre de Paris à Vienne pour le week-end. Si nous faisons abstraction un instant des remous géopolitiques du siècle passé, nous ne sommes pas plus libres que les générations précédentes (personne ne les empêchait de se rendre à Vienne à pied), mais notre capacité d'action a en revanche augmenté de manière vertigineuse. "La liberté est une chose - dit Berlin (ibid., p. 50) - et les conditions de son exercice une autre. » Nous pouvons également illustrer cela à l'aide de la distinction plus récente entre liberté formelle et liberté réelle. Nos grands-parents étaient formellement libres d'aller faire du tourisme en Autriche, mais ils n'en étaient pas réellement libres, n'ayant pas la capacité (ou la puissance) de le faire. La capacité d'action est donc ici ce qui permet d'actualiser une liberté formelle ${ }^{6}$.

Pourquoi insister sur une telle distinction conceptuelle? Parce qu'elle est essentielle pour comprendre les conséquences sur la liberté des individus d'un retour en arrière en termes de capacité d'action. Elle permet de mettre au jour, après son aspect transgressif, une nouvelle caractéristique du rapport entre liberté négative et progrès technique. Comme nous l'avons vu, une innovation technique peut augmenter la capacité d'action des individus sans pour autant augmenter leur liberté. Elle se contente d'actualiser une liberté formelle déjà existante. À l'inverse, si pour une raison ou une autre l'usage de cette technique venait à être interdit, cette interdiction serait immanquablement interprétée comme une restriction de liberté. À raison, puisqu'une telle interdiction est une contrainte intentionnelle d'origine humaine et tombe par là même sous le coup de la définition classique de la liberté négative ${ }^{7}$. Berlin dit bien que je ne suis pas libre "si je suis empêché par autrui de faire ce que sinon je pourrais faire » (cité par Skinner, 2002, p. 22). En nous en tenant strictement à ce cadre, nous pouvons donc dire que ne plus pouvoir prendre l'avion est une perte de liberté si c'est le fait d'une législation, mais n'est que le

\footnotetext{
6 Suivant en cela Skinner (2002), il faut cependant prendre garde au fait que la distinction entre liberté et capacité d'agir n'est pas exactement équivalente à la distinction entre liberté formelle et liberté réelle. Alors que les deux premières notions sont incommensurables - celui qui n'a pas la capacité d'agir ne peut pas être dit libre ou non-libre (c'est le point de vue de Berlin) -, la seconde distinction pourrait être grossièrement résumée sous la forme de l'équation «liberté formelle + capacité d'agir = liberté réelle». Dans ce cas, un aveugle peut être dit formellement libre de lire. Je suivrai dans la suite de mon raisonnement la position de Berlin.

7 Définition qui, rappelons-le, a structuré une grande partie du champ de la réflexion politique moderne. L'essai d'I. Berlin Two Concepts of Liberty, intégrant cette même conception de la liberté, a été qualifié $\mathrm{d}^{\prime}$ " essai le plus influent de la philosophie politique anglophone contemporaine » par plusieurs penseurs (Skinner, 2002, p. 16).
} 
simple retour à notre incapacité d'agir antérieure si c'est le fait d'une limite naturelle, comme par exemple l'épuisement des réserves de pétrole. On verrait mal, en effet, un gouvernement être traité d'autoritaire et de liberticide parce qu'il ne fournit plus à ses citoyens l'accès à une ressource énergétique devenue inexistante. Il y a donc une asymétrie entre la facilité qu'ont nos sociétés à acquérir de nouvelles capacités (une nouvelle puissance) et la difficulté qu'elles ont à en limiter l'usage, compte tenu de la sacralisation de la liberté négative qui caractérise les démocraties libérales. Or, c'est bien la puissance d'action associée à nos technologies qui pose problème aujourd'hui dans notre relation à l'environnement.

Tout le drame de la crise écologique globale à laquelle nous faisons face est qu'elle ne se résoudra pas - c'est à souhaiter - par une finitude de fait (du moins pas tant que nous n'aurons pas réellement touché aux limites de la planète), mais par une limitation volontaire de l'éventail de nos actions. La finitude des Anciens relevait du domaine de l'incapacité d'action. «Le cosmos antique bornait en effet tout autant les désirs que les possibilités d'action des citoyens » (Bourg et Whiteside, 2010). Nous, Modernes, avons des moyens techniques qui, associés à une métaphysique de l'illimité, sont propres à épuiser la planète. La finitude nouvelle qui nous est dévoilée par les sciences de l'environnement se traduira en nouvelles contraintes intentionnelles et d'institution humaine. Elles relèveront donc du domaine de la restriction de liberté, ce qui est loin d'être socialement acceptable. Nous pouvons ainsi voir dans le projet baconien d'émancipation par la technique le mouvement centrifuge qui étend sans cesse notre capacité d'action et dans la liberté négative du libéralisme classique le cran d'arrêt qui rend difficile tout retour en arrière.

\section{Liberté et propriété : théorie de l'individualisme possessif chez Locke et Nozick}

Pour Locke, l'Homme est libre dans le sens où il est le propriétaire de lui-même, ce qui lui donne un droit naturel au produit de son travail. Cette relation particulière entre propriété et liberté chez les libéraux classiques a été ramassée par Macpherson (2004) sous le nom de «théorie politique de l'individualisme possessif », ce qu'il résume de la manière suivante (ibid., p. 19) : «L'individu, pense-ton, n'est libre que dans la mesure où il est propriétaire de sa personne et de ses capacités. Or, l'essence de l'homme, c'est d'être libre, indépendant de la volonté d'autrui, et cette liberté est fonction de ce qu'il possède. » Il y a ici une relation dialectique entre liberté et propriété, la première étant nécessaire à la seconde et inversement. Dans la mesure où l'exploitation et l'appropriation des ressources naturelles sont en lien direct avec la problématique environnementale, il convient de s'intéresser de plus près à cet individualisme possessif dont Macpherson fait le soubassement idéologique du libéralisme.

Les réflexions sur le partage des biens dans les textes fondateurs et la différence d'interprétation du milieu naturel à l'état de nature que l'on peut noter entre Hobbes (la rareté) et Locke (l'abondance) sont assez instructives. Si Hobbes ne nous dit pas grand-chose de la nature des origines, il précise toutefois que la compétition est le premier motif qui pousse les hommes à se nuire mutuellement. «Et donc, si deux humains désirent la même chose, dont ils ne peuvent cependant jouir l'un et l'autre, ils deviennent ennemis et, pour parvenir à leur fin [...], ils s'efforcent de s'éliminer ou de s'assujettir l'un l'autre » (Hobbes, 2000, p. 222). La nature de l'homme, poursuit Hobbes, le pousse à acquérir par la force les biens nécessaires à sa propre conservation et c'est de la rareté que naît cette rivalité.

Mais, déjà bien avant cette saturation du monde, la recherche du profit associée à celle de la sécurité et de la réputation engendre la guerre de tous contre tous, d'où la nécessité d'un pouvoir fort et d'une liberté individuelle réduite pour assurer paix et sécurité. Il en va tout autrement chez Locke, pour qui ce n'est plus la mort mais la faim qui menace l'individu (Manent, 1997, p. 93) ${ }^{8}$. « [...] Les hommes possèdent un droit à leur propre préservation, et par conséquent à la nourriture, à la boisson et à toutes les autres choses que la nature offre pour leur subsistance » (Locke, 1994, § 25). De là découle le droit de s'approprier les fruits de la nature pour ses propres besoins. C'est le travail (la cueillette, la chasse et la mise en culture du sol) qui fonde ce droit de propriété. À l'inverse de chez Hobbes, cela n'occasionne aucun problème puisque le postulat de départ de Locke (ibid., §31) est non plus la rareté mais l'abondance:

«Donc, quand on considère l'abondance des provisions naturelles qu'il y eut pendant longtemps dans le monde ainsi que le petit nombre des consommateurs ; mais quand on voit aussi la faible part de ces provisions à laquelle l'industrie d'un seul homme pouvait s'étendre pour l'accumuler au préjudice des autres, surtout lorsqu'il se tenait dans les limites, fixées par la raison, de ce qui pouvait servir à son usage, il ne pouvait y avoir alors que peu de place pour les querelles et les contestations à propos d'une propriété ainsi établie. »

De même pour la terre, qui, après les «provisions naturelles », est la seconde catégorie de biens susceptibles d'appropriation (ibid., §33; cf. aussi § 36) :

«Cette appropriation d'une parcelle de terre, par le biais de sa mise en valeur, ne causait de préjudice à aucun homme, puisqu'il en restait suffisamment, et d'aussi bonne qualité ; plus même que ceux qui n'étaient pas encore pourvus ne pouvaient en utiliser. »

8 Les développements qui suivent reprennent en grande partie l'interprétation de Macpherson (2004) et les éclaircissements de Manent (1997) sur le libéralisme des origines. 
Nulle interférence, donc, entre les droits individuels à l'état de nature. Nous sommes là dans une situation diamétralement opposée à la conception hobbésienne de la condition naturelle de l'homme. À la rareté chez Hobbes, correspond un pouvoir fort et absolu; à l'abondance de Locke, un individu libre et un pouvoir régulateur chargé avant tout de protéger les droits du citoyen. Mais cette conclusion n'est pas aussi directe qu'il n'y paraît, car Locke pose deux limites claires à l'usage des biens naturels. La première est de s'en tenir à la quantité nécessaire à ses propres besoins pour éviter le gaspillage (§31), la seconde est d'en laisser suffisamment pour les autres ( $\$ 27$ et § 33). La modération est donc dictée par un souci de respect de la création et de partage. Sage précaution qui, malgré un contexte de profusion, laisse poindre un souci de la finitude chez le philosophe. Mais ce souci sera bien vite démenti, car, comme le note Manent (1997, p. 98), « toute la démarche de Locke consiste à abolir les deux limites qu'il a d'abord posées ».

C'est l'argent qui permettra de dépasser la première de ces deux limites, puisque, étant incorruptible, il permet, en les échangeant contre de la monnaie, de conserver de manière permanente la valeur des fruits qui ont été cueillis mais n'ont pas été consommés (Locke, 1994, § 46).

Dans les parties du monde fort peuplées et qui ont introduit l'usage de l'argent (l'Angleterre), Locke note toutefois que l'abrogation de cette première limite peut conduire à une raréfaction des terres (Locke, 1994, $\S 45$; Macpherson, 2004). Cela est cependant sans conséquence, car, par sa productivité, le travail humain augmente le capital naturel plus qu'il ne l'appauvrit. La seconde limite, consistant à faire preuve de modération pour laisser aux autres la possibilité de jouir à leur tour des fruits de la terre, est ainsi rendue nulle elle aussi. «C'est donc le travail qui donne à une terre la plus grande partie de sa valeur, et sans lui elle ne vaudrait pratiquement rien ; c'est à lui que nous devons la plus grande part des produits de la terre qui nous sont de quelque usage [...]» (Locke, 1994, § 43). Grâce à l'argent et à la productivité du travail, la rareté locale et momentanée des terres peut donc être aisément dépassée. Par ailleurs, il reste bien assez de terres encore sous-exploitées dans les autres régions du monde (en l'occurrence en Amérique) [ibid., § 41]. Pour ces raisons, Manent en conclut, suivant Macpherson, que, chez Locke, «l'individu a un droit naturel à une propriété qui n'a pas de limites naturelles » (Manent, 1997, p. 99 ; cf. Locke, 1994, § 49-50). C'est donc tout bonnement l'activité productive qui donne potentiellement à tous les hommes la possibilité d'actualiser leur droit fondamental à la propriété.

La théorie de Macpherson a été l'objet de critiques, tant au niveau de la pertinence de l'individualisme possessif concernant le XVII ${ }^{\mathrm{e}}$ siècle, que de l'interprétation détaillée des différents auteurs. L'idée, notamment, d'un droit à l'appropriation et à la consommation illimitée chez Locke a été remise en cause, dans la mesure où celle-ci est soumise à un devoir de charité envers les plus indigents (Miller, 1982; Tully, 1993). James Tully situe plutôt l'émergence de théories de l'appropriation illimitée au XVIII ${ }^{\mathrm{e}}$ siècle. Mais, au-delà des controverses interprétatives, la plupart des commentateurs s'accordent sur la valeur heuristique du concept d'individualisme possessif et sur sa pertinence pour problématiser certains aspects du libéralisme moderne et contemporain (Miller, loc. cit.; Tully, op. cit. ; Savidan, 2004). Ce qui nous importe ici est de voir que, chez Locke déjà, une certaine conception du rapport de l'homme à la nature prévalait et que la productivité du travail, sinon l'abondance de la nature, était au fondement de sa théorie de la propriété.

\section{Débats contemporains}

Si l'individualisme possessif n'est pas le reflet exhaustif des sociétés contemporaines, il est assurément un élément structurant important des démocraties libérales. L'illustration contemporaine la plus paradigmatique de ce principe se trouve dans l'œuvre du célèbre philosophe politique américain Robert Nozick, d'obédience libertarienne, c'est-à-dire néolibérale. Dans son livre Anarchie, État et utopie (Nozick, 2008), il accorde à la propriété de soi une importance centrale, faisant découler de celle-ci un droit légitime à la propriété des fruits de son travail. Les droits de propriété accordés aux individus sur les biens acquis par l'exercice de leurs facultés sont absolus et exclusifs, une simple taxation des revenus étant assimilée par Nozick à du travail forcé. Tous les transferts de propriété justes (en l'occurrence les transactions marchandes) donnent lieu à des droits de propriété légitimes si la situation de départ est elle-même juste.

Nozick en vient donc à s'interroger sur le critère qui rend légitime la première appropriation des biens naturels et cherche appui pour cela chez Locke. La fameuse clause lockéenne définissant la légitimité de l'appropriation des ressources du monde extérieur : en laisser «en quantité et en qualité suffisante » pour autrui, est interprétée de manière minimaliste. Toute appropriation initiale est juste si elle ne détériore par les conditions de vie de qui que ce soit. Un individu peut donc acquérir des droits absolus sur une quantité potentiellement illimitée de ressources si cela laisse la situation des autres individus meilleure ou inchangée. Cette théorie, dont on voit bien en quoi elle est un «individualisme possessif », permet à Nozick de justifier une politique de l'État minimal (pas de redistribution) et du libre marché (justice intrinsèque de l'échange marchand).

On retrouve ici, formulée de manière plus contemporaine, une justification de l'appropriation privée illimitée, les situations contrevenant à la clause lockéenne 
devant être, selon l'auteur libertarien, particulièrement rares. La propriété privée permet en effet d'augmenter la production en concentrant les ressources dans les mains les plus efficaces et en encourageant la prise de risque. Les personnes n'ayant plus accès à la propriété des ressources naturelles voient ainsi ce désavantage compensé par l'amélioration de la situation économique générale. Par ailleurs, le mécanisme des prix rend peu probable l'accaparement par une même personne de l'intégralité d'une ressource. Nozick (2008, p. 227) en conclut que «le fonctionnement libre d'un système de marché ne trahira pas véritablement la clause de Locke». Cette clause, qui seule peut limiter le droit d'appropriation, ne s'applique en définitive qu'aux cas de catastrophe imminente, comme celui d'un assèchement des puits dans un désert, ou à des cas assez théoriques (aux yeux de Nozick) d'une ressource nécessaire à l'existence devenue particulièrement rare (exemple de l'île déserte, pp. 224 à 226). Nous retrouvons donc ici implicitement les présupposés de l'abondance et de l'augmentation continue de la productivité, déjà identifiés chez Locke, dans la manière dont Nozick interprète le critère théorique qu'il a lui-même formulé.

Or, ce que nous apprennent aujourd'hui les sciences de l'environnement est justement qu'une multiplication des raretés est à l'œuvre à l'échelle de la planète (la finitude). Cela a pour effet d'augmenter singulièrement le nombre de cas dans lesquels la clause de Locke entre en application, allant à l'encontre même des finalités auxquelles Nozick destinait sa théorie. Il convient aujourd'hui non seulement de prêter attention à la raréfaction des ressources de première nécessité (Nozick pensait en premier lieu à l'eau potable; nous pourrions cependant en ajouter plusieurs dont les terres arables, la stabilité du climat, la qualité de l'air, etc.), mais également à l'usage qui est fait de ces ressources. Mon droit absolu sur le pétrole que je possède m'autorise-t-il à le brûler et à participer ainsi au changement climatique? Posséder une parcelle m'autorise-t-il à la polluer à volonté ? Au regard des problèmes écologiques actuels, on peut légitimement se demander si l'idée de droit de propriété absolu sur une ressource a encore du sens. En sus du fait que l'on puisse vouloir la rejeter pour des raisons d'incohérence théorique ou de justice sociale (Kymlicka, 2003), les conclusions que Nozick tire de sa théorie du droit de propriété se tournent en leur contraire dès lors que l'on postule la finitude intrinsèque des ressources naturelles, et non plus leur abondance. Cet exemple montre à quel point la théorie politique est dépendante de présupposés sur l'état du monde, présupposés qui sont en train de changer radicalement. Il y a dès lors fort à parier que la conception de la liberté sous-tendue par l'individualisme possessif soit remise en question par l'état d'une planète aux ressources toujours plus rares. La littérature environnementale en fournit de nombreux exemples.
Si la monnaie est aux yeux de Locke le moyen de conserver les denrées naturelles périssables, elle apparaît aujourd'hui de plus en plus souvent comme le principe abstrait détournant notre attention des stocks de ressources naturelles et responsable de leur épuisement. Comme l'expliquent Jancovici et Grandjean (2009, p. 85), «nous confondons en permanence les réalités (les ressources) et les symboles ou conventions (les prix). Nous ne voyons pas que l'argent n'est évidemment pas la richesse matérielle, même s'il sert d'étalon de mesure de cette richesse » (cf. aussi Calame et Lalucq, 2009). On pourra rétorquer, à l'instar de Nozick - qui ne fait ici que suivre la tradition du libéralisme économique -, que les mécanismes de marché répondent à ce problème par le signal-prix, qui est indicatif de la rareté d'une ressource. Un marché autorégulé ne fonctionne cependant que dans un contexte d'immédiateté ou de spéculation, alors que la situation actuelle demande au contraire de prendre des décisions, qualitatives autant que quantitatives, sur la préservation de certaines ressources en anticipant la rareté. Le marché ne dit rien, par exemple, sur le seuil de renouvellement des réserves halieutiques - déjà largement dépassé pour certaines espèces (Cury et Miserey, 2008) - ni sur la capacité de charge d'un écosystème. Il est aveugle aux effets de seuil et ne dit rien non plus de la valeur morale ou patrimoniale des êtres naturels (Arrow et al., 1995 ; Tinbergen et Hueting, 1991). Les économistes, Arthur Pigou en tête, se sont par ailleurs rendu compte depuis bien longtemps que le système économique laissait dans l'ombre de nombreux facteurs environnementaux tels que les pollutions ou les problèmes liés aux flux de matières, et en particulier le changement climatique. Or, le terme de finitude ne renvoie pas uniquement à l'idée d'un stock limité de biens naturels, mais également à la capacité limitée des écosystèmes à supporter les dérèglements fonctionnels qui leur sont imposés par les activités humaines (Rockström et al., 2009).

Quant à la thèse de l'abondance, nul besoin de grandes démonstrations pour prouver ses limites à l'heure du pic pétrolier, de la raréfaction des métaux semi-précieux, de l'affaissement des ressources halieutiques, des crises hydriques à répétition et de la ruée des pays émergents sur les terres arables (pour une bonne synthèse, cf. Science et vie, 2008; Le Monde, 2009 et 2010; Laherrère, 2011). La finitude est une réalité avec laquelle il faudra désormais composer et qui semble craindre particulièrement les situations de libre accès. En 1968, dans son fameux article « The tragedy of the commons », Garrett Hardin avait déjà exposé de manière magistrale la difficulté de gérer les biens communs dans un contexte de libre accès des acteurs. Le bénéfice de l'exploitation d'un bien commun par un individu (par exemple défricher une parcelle de forêt primaire) est pour lui toujours supérieur au désavantage qu'il provoque (la perte du service de régulation climatique offerte par cette parcelle 
de forêt), puisque ce dernier est partagé entre tous les bénéficiaires du bien commun. La conclusion est donc sans appel : «La liberté, dans les communaux, apporte la ruine à tous. » Et il s'ensuit naturellement que «nous devons réexaminer nos libertés individuelles pour voir lesquelles sont défendables » (Hardin, 1968, p. 1244, traduction de l'auteur). Les théories de Locke (1994) et de Nozick (2008) sont des théories de l'appropriation individuelle en situation de libre accès, dont le but est de promouvoir la liberté individuelle par le biais des droits à la propriété de soi et des ressources (Vallentyne, 2009). Mais, comme nous l'avons vu, leur propre critère de justice (la clause lockéenne) conduit à un affaiblissement du droit à l'appropriation en situation de rareté ou en l'absence de croissance économique. Une telle situation est celle que les sciences de l'environnement nous dévoilent peu à peu (Jackson, 2010). On ne peut dès lors qu'en inférer une limitation de la liberté d'action des individus au sens que lui donne le paradigme de l'individualisme possessif. L'appropriation et, par extension, l'usage des ressources ne peuvent plus se fonder ni sur la libre initiative ni sur le libre marché. Ils doivent être l'objet d'une gestion collective et différenciée tenant compte de la nature, de la qualité et de la quantité des ressources.

Nous avons vu avec Nozick la relation intime qui lie la conception moderne de la liberté et l'idée d'un monde abondant en ressources. Mais le débat contemporain sur les problèmes environnementaux fait apparaître des questions d'un type nouveau : Jusqu'où la consommation et l'accumulation matérielles d'une société peuvent-elles s'exercer au mépris des dégradations environnementales? Qu'en est-il de la multiplication des trajets en avion, sachant que chacun d'eux émet jusqu'à dix fois plus de $\mathrm{CO}_{2}$ que certains habitants de la planète durant toute une année ? La production d'agrocarburants peut-elle se faire au détriment de cultures vivrières au nom de la liberté du marché? Etc.

En raison des limites chaque année plus saillantes des ressources de notre planète, les fondements du libertarisme s'effondrent. De manière plus générale, c'est même certaines notions-clés de la démocratie libérale qui sont aujourd'hui à repenser, en première position desquelles cette dialectique entre liberté et droit à l'accumulation illimitée.

\section{Conclusion}

Dans les parties qui précèdent, il a été vu que :

- le concept de liberté négative, associé à l'idéologie du progrès, permet une extension illimitée des capacités $\mathrm{d}^{\prime}$ 'action humaines tout en rendant difficile un retour en arrière ;

- le fondement philosophique de la doctrine libérale (du moins dans sa variante néolibérale) ouvre la porte à l'accumulation illimitée, intimement liée à la liberté des individus, sur une base qui ne peut plus aujourd'hui être considérée comme valide.

Ces conclusions sont les deux faces visibles d'une même pièce que Bourg et Whiteside (2009) ont appelé "métaphysique de la liberté moderne », et qui toutes deux relèvent d'une logique de la transgression. Dans les deux cas, on décèle une volonté de dépassement des bornes imposées à l'humain. Nous avons affaire à la négation de la finitude humaine - c'est-à-dire de l'idée qu'il existe des limites intrinsèques à la capacité d'action des humains - dans le premier cas et à la négation de la finitude de la nature dans le second. Ces deux dimensions sont inextricablement liées, dans la mesure où une capacité d'action accrue implique la possibilité d'un impact accru sur la nature et ses ressources.

La reconnaissance de ce fait fut, à compter des années 1950, suffisamment puissante pour engendrer divers courants qui ont cherché à repenser les bases de la vie en commun en y intégrant la question du rapport à l'environnement. L'écologie politique, par exemple, incarne l'un des courants les plus vigoureux et les plus inventifs à cet égard. Elle suppose cependant une révolution radicale de notre manière de vivre ensemble, organisée autour de la communauté, et une conception de l'épanouissement humain séparé de l'accumulation matérielle - une véritable anthropologie de l'autolimitation s'appuyant sur une «norme du suffisant» (Gorz, 1992). Quant à l'autoritarisme assumé de Hans Jonas, les totalitarismes du $X X^{\mathrm{e}}$ siècle $\mathrm{n}^{\prime}$ ont que trop bien montré les tragédies sur lesquelles il peut déboucher. Dans le cadre démocratique contemporain, la question de la liberté individuelle et de ses relations avec la protection de l'environnement reste donc entière. S'il est vrai que, dans l'histoire de nos sociétés, la liberté des individus n'a jamais été totale (ce serait nier le principe même de la vie en société) et que la liberté négative a pu connaître des retours en arrière dans certains domaines, il ne faut pas oublier, en revanche, que Locke écrivait contre l'absolutisme et contre l'arbitraire. On mesure mal la dette que nous avons vis-à-vis des idéaux du libéralisme politique, qui nous ont légué les Droits de l'homme et l'État de droit. C'est bien toute la difficulté de la tâche. Les droits humains ont toujours été le lieu de contradictions. L'intégration des questions environnementales dans le débat en fait apparaître de nouvelles, qui ne manqueront sans doute pas de donner lieu à leur relecture. La question des relations entre la liberté humaine et la prise en compte de la donne environnementale est donc centrale pour l'évolution de nos société vers un fonctionnement plus éco-compatible, sans pour autant sacrifier les valeurs démocratiques. Elle ne se limite d'ailleurs pas aux deux aspects qui ont été brièvement explorés dans cet article et il ne fait nul doute que ce thème sera à l'avenir un élément-clé des débats sur la transition écologique. 


\section{Références}

Arrow, K., Bolin, B., Costanza, R., Daspupta, P., Folke, C., Holling, C.S., Jansson, B.-O., Levin, S., Mäler, K.-G., Perrings, C., Pimentel, D., 1995. Economic growth, carrying capacity, and the environment, Science, 268, 5210, 520-521.

Berlin, I., 1988 [éd. orig. 1969]. Éloge de la liberté, Paris, CalmannLévy.

Bourg, D., 1997. Nature et technique : essai sur l'idée de progrès, Paris, Hatier.

Bourg, D., 2009. L'impératif écologique, Esprit, 360, 59-71.

Bourg, D., Whiteside, K., 2009. Pour une démocratie écologique, La Vie des idées : http://www.laviedesidees.fr/ Pour-une-democratie-ecologique.html (consulté le 4 août 2010).

Bourg, D., Whiteside, K., 2010. Vers une démocratie écologique : le citoyen, le savant et le politique, Paris, Le Seuil.

Calame, P., Lalucq, A., 2009. Essai sur l'economie, Paris, Charles Léopold Mayer.

Constant, B., 1997 [éd. orig. 1814-1819]. Écrits politiques, Paris, Gallimard.

Cury, P., Miserey, Y., 2008. Une mer sans poissons, Paris, CalmannLévy.

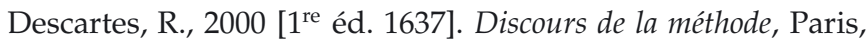
Flammarion.

Felli, R., 2008. Les Deux Âmes de l'écologie : une critique du développement durable, Paris, L'Harmattan.

Ferry, L., 2002 [1' éd. 1992]. Le Nouvel Ordre écologique : l'arbre, l'animal et l'homme, Paris, Grasset.

Gauchet, M., 2010. Remarques préliminaires sur la politique écologique, in Bourg, D., Papaux, A. (Eds), Vers une société sobre et désirable, Paris, PUF, 163-171.

Gorz, A., 1978 [1 $1^{\text {re }}$ éd. 1975, publiée sous le nom de Michel Bosquet]. Écologie et politique, Paris, Le Seuil.

Gorz, A., 1992. L'écologie politique entre expertocratie et autolimitation, L'Écologie, ce matérialisme historique, Actuel Marx, $12,15-29$.

Grosse, F., 2010. Le découplage croissance/matières premières, Futuribles, 365, 99-124.

Hardin, G., 1968. The tragedy of commons, Science, 162, 3859, 1243-1248.

Hobbes, T., 2000 [éd. orig. 1651]. Léviathan, Paris, Gallimard.

Hulot, N., et le comité de veille écologique, 2009. Évolution : chapitre 2. Première contribution pour une refondation écologique et solidaire de nos sociétés : www.fondation-nature-homme. org [nos actions ; nos publications ; toutes nos publications] (consulté le 4 août 2010).

Illich, I., 2003 [1 $1^{\text {re }}$ éd. 1973]. La Convivialité, Paris, Le Seuil.

Jackson, T., 2010. Prospérité sans croissance : la transition vers une économie durable, Bruxelles, De Boeck.

Jancovici, J.-M., Grandjean, A., 2006. Le Plein s'il vous plaît : la solution au problème de l'énergie, Paris, Le Seuil.

Jancovici, J.-M., Grandjean, A., 2009. C'est maintenant! 3 ans pour sauver le monde, Paris, Le Seuil.
Jonas, H., 1990 [éd. orig. 1979]. Le Principe responsabilité : une éthique pour la civilisation technologique, Paris, Cerf.

Kymilicka, W., 2003 [éd. orig. 1990]. Les Théories de la justice: une introduction, Paris, La Découverte.

Laherrère, J., 2011. Les perspectives pétrolières et gazières, Futuribles, 373, 5-28.

Lebeau, A., 2005. L'Engrenage de la technique : essai sur une menace planétaire, Paris, Gallimard.

Lebeau, A., 2008. L'Enfermement planétaire, Paris, Gallimard.

Locke, J., 1994 [éd. orig. 1690]. Le Second Traité du gouvernement: essai sur la véritable origine, l'étendue et la fin du gouvernement civil, Paris, PUF.

Longeaux, N. de, 2009. La Nature et la norme : la philosophie politique contemporaine face aux questions écologiques, Paris, L'Harmattan.

Macpherson, C.B., 2004 [éd. orig. 1962]. La Théorie politique de l'individualisme possessif : de Hobbes à Locke, Paris, Gallimard.

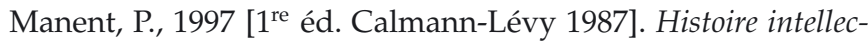
tuelle du libéralisme : dix leçons, Paris, Hachette.

Miller, D., 1982. The Macpherson version, Political Studies, 30, $1,120-127$.

Monde (Le), 2009. Bilan de la planète, hors-série.

Monde (Le), 2010. Bilan de la planète, hors-série.

Nozick, R., 2008 [éd. orig. 1974]. Anarchie, État et utopie, Paris, PUF.

Rockström, J., et al., 2009. A safe operating space for humanity, Nature, 461, 282, 472-475.

Rosanvallon, P., 2010. Le souci du long terme, in Bourg, D., Papaux, A. (Eds), Vers une société sobre et désirable, Paris, PUF, 151-162.

Rousseau, J.-J., 1996 [1 $1^{\text {re }}$ éd. 1754]. Discours sur l'origine et les fondements de l'inégalité parmi les hommes, précédé de Discours sur les sciences et les arts, Paris, Librairie générale française.

Savidan, P., 2004. Postface : L'individu et ses propriété, in Macpherson, C.B., La Théorie politique de l'individualisme possessif : de Hobbes à Locke, Paris, Gallimard, 534-583.

Science et vie, 2008. Construire un monde durable, hors-série 243.

Skinner, Q., 1991. Thomas Hobbes et le vrai sens du mot liberté, Archives de philosophie du droit, 36, 191-215.

Skinner, Q., 2002. Un troisième concept de liberté au-delà d'Isaiah Berlin et du libéralisme anglais, Acutel Marx, 32, 15-49.

Tully, J., 1993. An Approach to Political Philosophy: Locke in Contexts, Cambridge, Cambridge University Press.

The Good Society, 17, 2, 2008. Actes du symposium Green Constitutionalism: http://muse.jhu.edu/journals/good_society/toc/ gso.17.2.html (consulté le 4 août 2010).

Tinbergen, J., Hueting, R., 1991. GNP and market prices: Wrong signals for sustainable economic success that mask environmental destruction, in Goodland, R., Daly, H., El Serafy, S., von Droste, B. (Eds), Environmentally Sustainable Economic Development: Building on Brundtland, Paris, Unesco, 51-57.

Vallentyne, P., 2009. Left-Libertarianism and liberty, in Christiano, T., Christman, J. (Eds), Debates in Political Philosophy, Oxford, Wiley-Blackwell, 137-151. 